\title{
Puerarin inhibits the osteoclastogenesis by inhibiting RANKL-dependent and - independent autophagic responses
}

\author{
Guoyou Zhang ${ }^{1,2,3+}$, Yu Wang ${ }^{1,4+}$, Guoke Tang ${ }^{5}$ and Yuanzheng Ma, ${ }^{1,2^{*}}$ (D)
}

\begin{abstract}
Background: Puerarin exerts therapeutic effect on osteoporosis due to its inhibitory effect on the formation of osteoclasts. Puerarin is also widely established as an autophagy inhibitor. The study aimed to investigate the significance of autophagy in Puerarin-treated osteoclast formation.

Methods: Osteoclast precursors (OCPs) derived from bone marrow-derived macrophages (BMMs) were treated with Puerarin along with RANKL or without RANKL, and then the autophagic parameters of OCPs (including autophagic proteins, LC3 transformation, autophagosome or LC3-puncta) were observed through Western Blotting, Transmission Electron Microscopy and Immunofluorescence assays. Next, after using overexpression vectors of autophagic genes (Atg7, Atg5 and BECN1) to alter autophagy activity, OCP proliferation was measured by Ethynyl deoxyuridine (EdU) assays and Cell Counting Kit-8 (CCK-8) kit, and osteoclast differentiation was assessed by Tartrate-resistant acid phosphatase (TRAP) staining.

Results: The results showed that Puerarin could directly inhibit the autophagy and proliferation of OCPs. Importantly, overexpression of autophagic genes Atg5, Atg7 and BECN1 reversed Puerarin-inhibited OCP autophagy and proliferation. What's more, RANKL could promote the autography of OCPs, which was recovered by Puerarin treatment. Interestingly, different from single-Puerarin treatment, we found that in the presence of RANKL, only BECN1 overexpression significantly reversed Puerarin-inhibited osteoclast differentiation and OCP autophagy.

Conclusion: In conclusion, Puerarin could inhibit the OCP autophagy in the presence or absence of RANKL, which blocked the OCP proliferation and osteoclast differentiation respectively. Moreover, BECN1 plays an essential role in Puerarin-inhibited osteoclastogenesis. Our study provides potential clue to further complete the intrinsic mechanism of Puerarin in treating osteoporosis.
\end{abstract}

Keywords: Puerarin, Osteoclast, Autophagy, BECN1, Osteoporosis

\section{Background}

Pueraria lobata is a leguminous plant in China, which is widely used in the treatment of cardiovascular diseases, diabetes, osteonecrosis and neurodegeneration [1]. As an extract from Pueraria lobate, Puerarin is a phytoestrogen with significant bone-protective effect. Its therapeutic

\footnotetext{
* Correspondence: MYZPLA8rd@163.com

${ }^{+}$Guoyou Zhang and Yu Wang contributed equally to this work.

'Department of Orthopedics, Southern Medical University, Guangzhou 510515, Guangdong, China

2Department of Orthopaedics, The 8th medical center of chinese PLA general hospital, No. 17 Heishanhu Road, Haidian District, Beijing 100091, China

Full list of author information is available at the end of the article
}

effect has been broadly reported in the treatment of osteoporosis. Cho et al. [2] found that the decrease in the femoral bone density of ovariectomized mice was inhibited after feeding Puerarin-containing diet for 4 weeks. Another study found that Puerarin could alleviate streptozotocininduced osteoporosis in rats via HDAC1/HDAC3 signaling [3]. In addition, Puerarin could also prevent bone loss in castrated male rats [4]. The inhibitory effect of Puerarin on osteoclasts matters a lot in its treatment of bone loss. Pueraria extracts are known to inhibit RANKL-stimulated osteoclastogenesis in the dose-dependent manner and to reduce bone resorption activity of osteoclasts [5]. Puerarin also reduced the formation of mature osteoclasts in RANKL-induced

(c) The Author(s). 2019 Open Access This article is distributed under the terms of the Creative Commons Attribution 4.0 International License (http://creativecommons.org/licenses/by/4.0/), which permits unrestricted use, distribution, and reproduction in any medium, provided you give appropriate credit to the original author(s) and the source, provide a link to the Creative Commons license, and indicate if changes were made. The Creative Commons Public Domain Dedication waiver (http://creativecommons.org/publicdomain/zero/1.0/) applies to the data made available in this article, unless otherwise stated. 
RAW264.7 cells [6]. In addition, Puerarin can also prevent lipopolysaccharide-stimulated osteoclastogenesis and bone loss [7]. However, the mechanism regarding the effect of Puerarin on inhibiting the osteoclastogenesis remains unclear.

Previous studies disclosed that the protective autophagy exerts an indispensable effect on the osteoclast formation as well as bone absorption activity of osteoclast [8-10]. In addition, a considerable number of studies suggested that Puerarin could regulate the autophagic activity. Some reports have shown that Puerarin upregulates autophagy [11-13], but some studies showed that it inhibits autophagy [14-16]. He et al. [14] suggested that Puerarin had neuroprotective effect against cerebral ischemia, which was related to the decrease of autophagic activity in neurons after its intervention. It was also reported that Puerarin could prevent rat brain from ischemia/reperfusion injury through repressing the autophagic response [15]. In addition, Puerarin pretreatment reduced the hypoxia/reoxygenation injury via inhibiting the Akt-autophagy signaling in the myocardium [16]. Therefore, whether the effect of Puerarin on inhibiting the osteoclastogenesis is by mediating the change of autophagic activity is worth further exploring.

Osteoclastogenesis consists of the proliferation and differentiation of OCPs. RANKL is a key inducing factor in osteoclast differentiation. Autophagy not only plays an important role in OCP proliferation [17], but also regulates OCP differentiation under RANKL intervention [8, 9]. Thus, we can clarify the overall significance of autophagy in Puerarintreated osteoclastogenesis by observing the effects of Puerarin on the autophagic activity of OCPs in the presence and absence of RANKL, respectively. During the autophagy response, a cytosolic form of LC3 (LC3I) forms membranebound LC3 (LC3II) by conjugating to phosphatidyl inositol. Thus, LC3 transformation and LC3 puncta are pivotal parameters for observing autophagic activity [18], including the osteoclastogenesis $[8,10,19,20]$. On the one hand, as important autophagy parameters, LC3 conversion rate and LC3 puncta number were upregulated by RANKL in OCP $[8,19]$; On the other hand, LC3 plays a significant role in the osteoclastogenesis. In this study, the detection of autophagic activity was focused on LC3-related indicators [10].

This study showed a role of Puerarin in inhibiting the OCP autophagy in the absence or presence of RANKL, which contributed to the reduction in OCP proliferation or OCP differentiation, respectively. Therefore, by clarifying the significance of Puerarin in the OCP autophagy, the present study revealed an autophagic mechanism underlying Puerarin-treated osteoclastogenesis for the first time.

\section{Methods}

\section{Reagents}

Recombinant M-CSF and RANKL were purchased from Peprotech (Rocky Hill, NJ, USA). Puerarin, E64d, Pepstatin
A (PEPS A) and TRAP staining kit were obtained from Sigma-Aldrich (St. Louis, MO, USA). Rabbit LC3B, Atg5, Atg7, Beclin1, and $\beta$-actin antibodies were purchased from Cell Signaling Technology (Beverly, MA, USA). Cell Counting Kit-8 (CCK-8) kit was obtained from Dojindo (Shanghai, China). The EdU kit was purchased from Roche (Mannheim, Germany). The SYBR Premix Ex TaqTM kit was from TakaRa (Tokyo, Japan). After dissolving in 1\% BSA, different working concentration of Puerarin $(0,10,25$, $50 \mu \mathrm{M})$ were prepared by complete $\alpha$-minimum Eagle's medium ( $\alpha$-MEM).

\section{Isolation and induction of OCPs}

4-8 week old littermate C57BL/6 J female mice were purchased from Slaccas Experimental Animal Centre (Shanghai, China). The mice were housed in a common environment in which the room temperature was $20 \sim 30^{\circ} \mathrm{C}$ and the humidity $60 \sim 80 \%$ and fed a general laboratory diet. The mice were sacrificed by cervical dislocation, and the tibia from mice were flushed using $\alpha$-MEM without serum. Bone marrow cells were incubated with aMEM containing 10\% FBS, penicillin $(100 \mathrm{U} / \mathrm{ml})$ and streptomycin $(100 \mathrm{mg} / \mathrm{ml})$ for $24 \mathrm{~h}$. Non-adherent cells were collected as Bone marrow-derived macrophages (BMMs). BMMs were induced to OCPs (adherent cells) after treatment with M-CSF $(20 \mathrm{ng} / \mathrm{ml})$ for 3 days as previously described $[21,22]$. Cells were cultured in the humidified atmosphere at $37{ }^{\circ} \mathrm{C}$ and containing $5 \% \mathrm{CO}_{2}$. The experimental protocols were approved by the Institutional Animal Care and Use Committee of Southern Medical University (No.44002100017774). To further elucidate the direct effect of Puerarin on the expression of autophagic proteins, cells were treated with Puerarin at different concentrations for $6 \mathrm{~h}$ without RANKL.

\section{Osteoclast differentiation assay}

OCPs $\left(1 \times 10^{5} /\right.$ well $)$ were incubated in 24-well plate in $\alpha$-MEM involving M-CSF (20 ng/ml) plus RANKL (100 $\mathrm{ng} / \mathrm{ml}$ ) supplemented with other relevant treatment for 5 days to induce mature osteoclasts. TRAP stainingpositive multinucleate cells (having more than three nuclei) were regarded as the differentiated osteoclasts.

\section{Cell proliferation assay}

Cell proliferation was evaluated using EdU assays and CCK-8 kit. In EdU assays, cell proliferation was quantified by observing EdU-positive cells, and in CCK-8 analysis, cell proliferation was measured by observing relative cell number. For EdU assays, OCPs $\left(1 \times 10^{5} /\right.$ well $)$ were cultured in 6-well plate, and treated with indicated treatment. Then, EdU assays were performed by using the EdU kit in accordance with the instruments. The results were collected and quantified under the Zeiss Photomicroscope (Carl Zeiss, Oberkochen, Germany) on the basis of at least 
ten random fields. For CCK- 8 analysis, OCPs were incubate in 96-well plate at a density of $1 \times 10^{4} /$ well, and then treated with different interventions. Next, cells were treated with the CCK- 8 reagents for $1 \mathrm{~h}$. Ultimately, the optical density at $450 \mathrm{~nm}$ (OD450) was observed by using Varioskan Flash reader (Thermo, MA, USA).

\section{Lentivirus infection}

Lentivirus encoding Atg5, Atg7 or BECN1 (including the corresponding control vector) were constructed by homologous recombination between expression vector (EXPuro-Lv105) and cDNA in 293 cells using the construction kit (GeneCopoeia, MD, USA) as previously described [23]. After 2 days, supernatants were collected and OCPs were incubated in the viral fluid containing $8 \mu \mathrm{g} / \mathrm{ml}$ polybrene at MOI 10 for 2 d. Transduced cells were selected by puromycin $(7.5 \mu \mathrm{g} / \mathrm{ml})$. The expression of viral genes was observed using qRT-PCR.

\section{Western blotting (WB) analysis}

The whole-cell lysate protein from cells (about $1 \times 10^{6}$ / well) with indicated interventions in 6-well plates were prepared. Lysates were packed into 10\% SDS-PAGE gels and polyvinylidene fluoride (PVDF) membranes were incubated with the antibodies against Atg5, Atg7, Beclin1, and LC3B, and $\beta$-actin after trarsmembrane. Horseradish peroxidase-linked secondary antibodies were used as secondary antibodies. Bands were visualized using a chemiluminescence system (Amersham Image 600, General Electric, MA, USA).

\section{Quantitative real-time PCR (qRT-PCR)}

The total RNA was extract and purified by the TRIzol method. Synthesis of cDNA and real-time quantitative PCR (qRT-PCR) measurements were performed as described previously [21]. The pre-designed primer sequences for qRT-PCR analysis were as following:

Cathepsin K (CTSK): 5'-GGAAGAAGACTCAC CAGAAGC-3' (forward) and 5'-GTC-ATATAGCCGC CTCCACAG-3' (reverse); Matrix metalloproteinase-9 (MMP-9):5' -CC-TGTGTGTTCCCGTTCATCT-3' (forward) and 5' -ACCCGAATCTAGTAAGGTCGC-3' (reverse); TRAP: 5'-GCTGGAAACCATGATCACCT-3' (forward) and 5'-TTGAGCCAGG-ACAGCTGAGT-3'

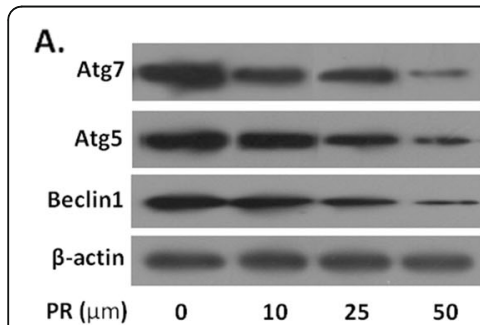

B.

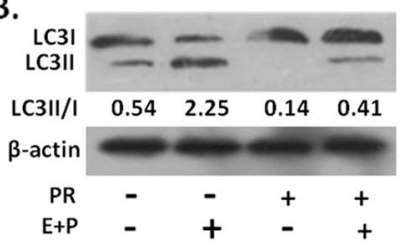

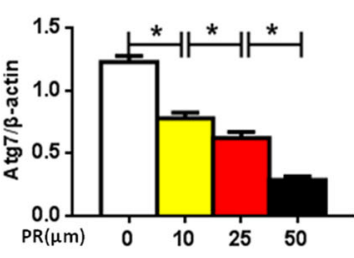

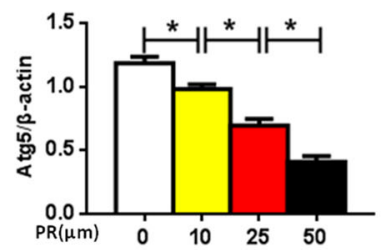

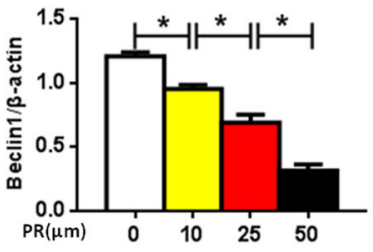

D.

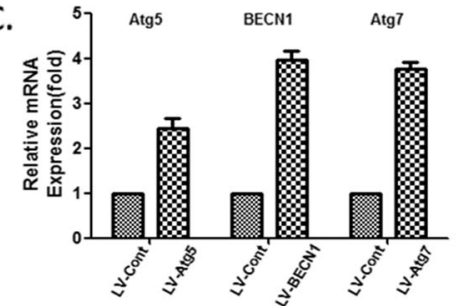

LV-BECN1+PR

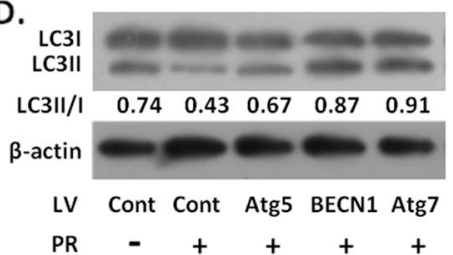

F.

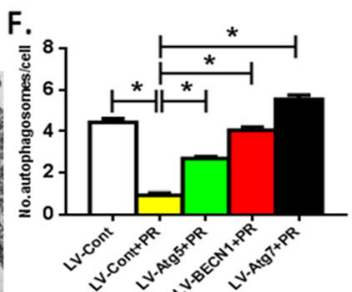

Fig. 1 Puerarin directly inhibits OCP autophagy. a The protein levels of Atg7, Beclin1 and Atg5 in the OCPs treated with different levels of Puerarin for $6 \mathrm{~h}$ without RANKL were measured by Western Blotting. Compared between Group $0 \mu \mathrm{M}$ and Group $10 \mu \mathrm{M}$, Group $10 \mu \mathrm{M}$ and Group $25 \mu \mathrm{M}$, Group $25 \mu \mathrm{M}$ and Group $50 \mu \mathrm{M}$, respectively, ${ }^{*} P<0.05$. b The ratio of LC3II/I in the OCPs treated with Puerarin (50 $\left.\mu \mathrm{M}\right)$ in the presence or absence of E64d + PEPS A for $8 \mathrm{~h}$ without RANKL. c mRNA expression of Atg5, BECN1 or Atg7 in the treated OCPs after infection with lentivirus encoding Atg5, BECN1 or Atg7 (LV-Atg5, LV-BECN1 or LV-Atg5) and the corresponding control vector (LV-Cont). d Viruses-transduced OCPs were treated with Puerarin $(50 \mu \mathrm{M})$ for $6 \mathrm{~h}$ without RANKL, and LC3II/I was detected by Western Blotting. e The autophagosomes (yellow arrows) in the viruses-transduced OCPs treated with Puerarin $(50 \mu \mathrm{M})$ for $24 \mathrm{~h}$ without RANKL were observed using TEM. Scale bar, $1 \mu \mathrm{m}$. $\mathbf{f}$ Statistical diagrams showed the quantitative results of autophagosomes from $\mathrm{E}$ (45 cells from three independent assays). Data are presented as mean $\pm \mathrm{SEM}$ from three independent experiments. Compared between Group LV-Cont and Group LV-Cont+PR, Group LV-Cont+PR and Groups LV-Atg5/BECN1/ Atg7 + PR, respectively, ${ }^{*} P<0.05$. PR, Puerarin; Cont, control groups; E, E64D; P, PEPS A 
(reverse); Atg7, 5' -GTTCGCCCCCTTTAATAGTGC-3' (forward) and 5'-TGAACTCCAACGTCAAGCGG-3' (reverse); Atg5, 5'-ATGCGGTTGAGG-CTCACTTTA3' (forward) and 5'-GGTTGATGGCCCAAAACTGG-3' (reverse); BECN1: $5^{\prime}$-CTAAGGCAGGCAGGAGGATG3' (forward) and 5'-GCTGGCCTCAA-GAGATCCAT - 3' (reverse); Cyclophillin A: 5' -CGAGCTCTGAGCAC 'TGGAGA-3' (forward) and 5'-TGG-CGTGTAAAGT CACCACC-3' (reverse).

qRT-PCR analysis was carried out by SYBR Premix Ex TaqTM kit and using ABI7500 analyzer (Thermo, MA, USA).

\section{Transmission Electron microscopy analysis}

Cells $\left(5 \times 10^{5}\right)$ were incubated on 6 -cm dishes as described above, followed by indicated treatments. The preparation of cell slices and subsequent staining were performed according to the protocol (Servicebio, Wuhan, Hubei, China) as previously described [19]. Then, the stained slices were observed under the 7700 transmission electron microscopy (Hitachi, Tokyo, Japan).

\section{Immunofluorescence assay}

Cells were incubated on $6-\mathrm{cm}$ dishes, and stimulated with different reagents. The treated cells $\left(1 \times 10^{6} /\right.$ tube $)$ were enriched in flow tubes, and then fixed by using $4 \%$ paraformaldehyde (PFA). Following perforation, cells were blocked using $1 \%$ bovine serum albumin (BSA), and incubated with the antibody targeting LC3B at $4{ }^{\circ} \mathrm{C}$ for $12 \mathrm{~h}$. Then, cells were stained with Cy3-labeled Goat Anti-Rabbit IgG for $1 \mathrm{~h}$. Next, cell suspensions were transferred to the adhesive slide. Thirty minutes later, the suspensions were removed, and the cells were counterstained using DAPI. Ultimately, the cells were observed and recorded under the fluorescence microscope (Olympus IX71, Tokyo, Japan). The cells with more than five LC3-punctas were regarded as LC3puncta positive cells [24, 25].

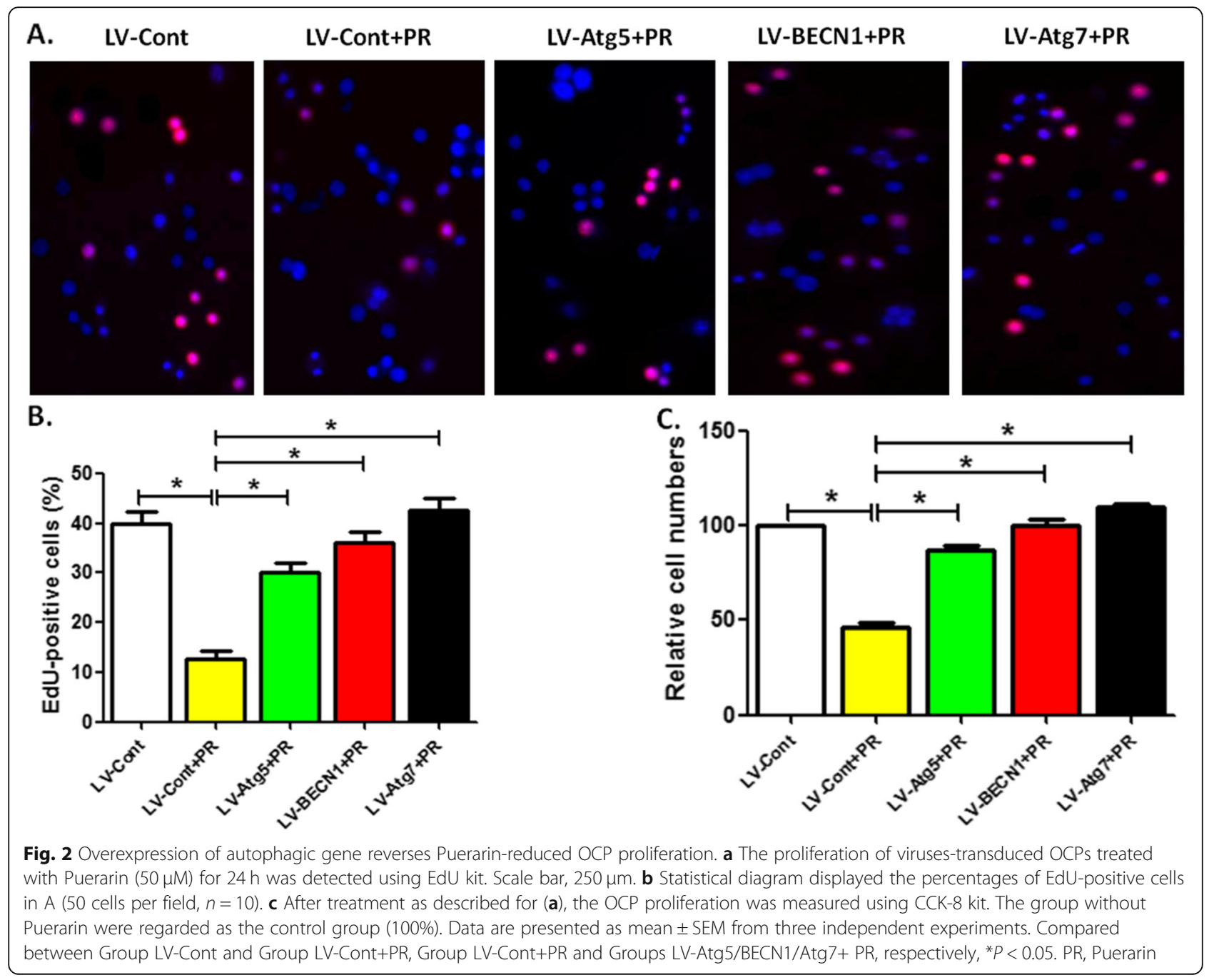




\section{Statistics}

The data are presented as mean + SEM. Statistical differences among groups were evaluated with one-way ANOVA analysis. Bonferroni test was used for Post Hoc Multiple Comparisons. Statistical significance was set at $P<0.05$. The statistical analyses were carried out using SPSS 19.0 software.

\section{Results}

Puerarin directly inhibited OCP autophagy

We first observed the direct effect of Puerarin on OCP autophagy without RANKL. The results showed that directly intervened by Puerarin, the expression of autophagic proteins Atg5, Atg7 and Beclin1 in OCPs decreased in a concentration-dependent manner (Fig. 1a). It could be seen that $50 \mu \mathrm{m}$ Puerarin had the strongest activity in inhibiting the expression of autophagic proteins (Fig. 1a). Thus, we chose this concentration of Puerarin for subsequent experiments. As shown in Fig. 1c-f, $50 \mu \mathrm{m}$ of Puerarin inhibited the LC3 transformation (defined as LC3II/I) and autophagosome formation in OCPs. Furthermore, LC3II/I was enhanced under the administration of PEPS A plus E64D (Fig. 1b), confirming that autophagy flux under this experimental system was smooth. Nonetheless, the inhibitory effect of Puerarin was reversed when Atg5, Atg7 or Beclin1 gene BECN1 were overexpressed by lentiviral transduction (Fig. 1c-f), and the effect of Atg7 was the most significant (Fig. 1d-f).

\section{Overexpression of autophagic gene reversed the reduced proliferation of OCPs by Puerarin}

Next, we assessed the effect of autophagic regulation on the proliferation of OCPs treated with Puerarin. EdU and CCK- 8 assay showed that after $24 \mathrm{~h}$ intervention, Puerarin at $50 \mu \mathrm{m}$ significantly inhibited the relative number of OCPs, which was recovered with the overexpression of Atg5, Atg7 or BECN 1 (Fig. 2a-c). Among them, Atg7 had the strongest recovery effect.

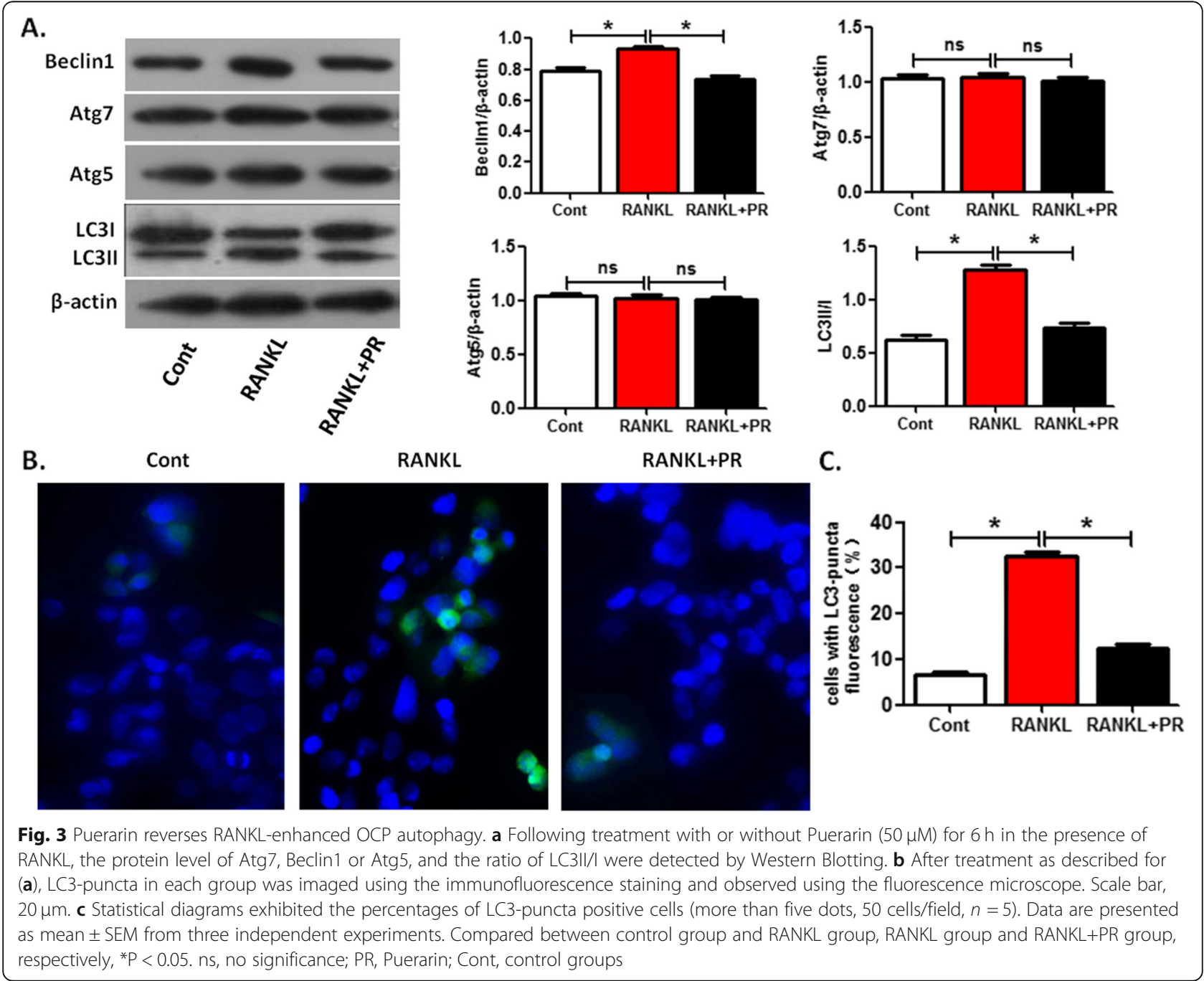


Puerarin reversed the enhanced effect of RANKL on OCP autophagy

The direct effect of Puerarin on regulating the OCP autophagy and subsequent OCP proliferation was documented. It is known that RANKL could promote the autophagy of OCPs $[26,27]$. Therefore, we further evaluated the effect of Puerarin on RANKL-regulated OCP autophagy. RANKL upregulated the expression of Beclin1, LC3 transformation and LC3 puncta formation in OCPs, but had no effect on the level of Atg5 and Atg7 (Fig. 3a-c). However, the effect of RANKL on promoting the above autophagic parameters of OCPs decreased with the supplement of $50 \mu \mathrm{m}$ Puerarin (Fig. 3a-c).

\section{BECN1 overexpression reversed Puerarin-inhibited OCP} autophagy and osteoclast differentiation in the presence of RANKL

Next, we observed the effects of the autophagic activity on RANKL-regulated OCP autophagy and osteoclast differentiation in the presence of Puerarin treatment. Under the intervention of RANKL, the LC3 transformation of OCPs inhibited by Puerarin was reversed by
BECN1 overexpression, but the overexpression of the other two autophagic genes Atg5 or Atg7 could not affect Puerarin-treated OCP autophagy (Fig. 4a, b). In addition, TRAP staining showed that the mature osteoclasts differentiated from co-inducement of RANKL and M-CSF decreased with the administration of $50 \mu \mathrm{m}$ Puerarin, which was recovered by BECN1 overexpression (Fig. 4c, d). Overexpression of Atg5 or Atg7 could not reverse the anti-osteoclastogenic effect of Puerarin (Fig. 4c, d). qPCR results revealed that the variation in the mRNA level of the three marker enzymes regarding the osteoclastogenesis was similar to TRAP staining results (Fig. 4e-g).

\section{Discussion}

As a phytoestrogen, Puerarin is known to inhibit the formation of osteoclasts, yet the detailed mechanism remains unclear, which raises an interesting scientific question for investigation. The effect of Puerarin on autophagic response has been verified. Here, we provide the first evidence demonstrating the effect of Puerarin on regulating the autophagic activity of OCPs. Combining up-to-date

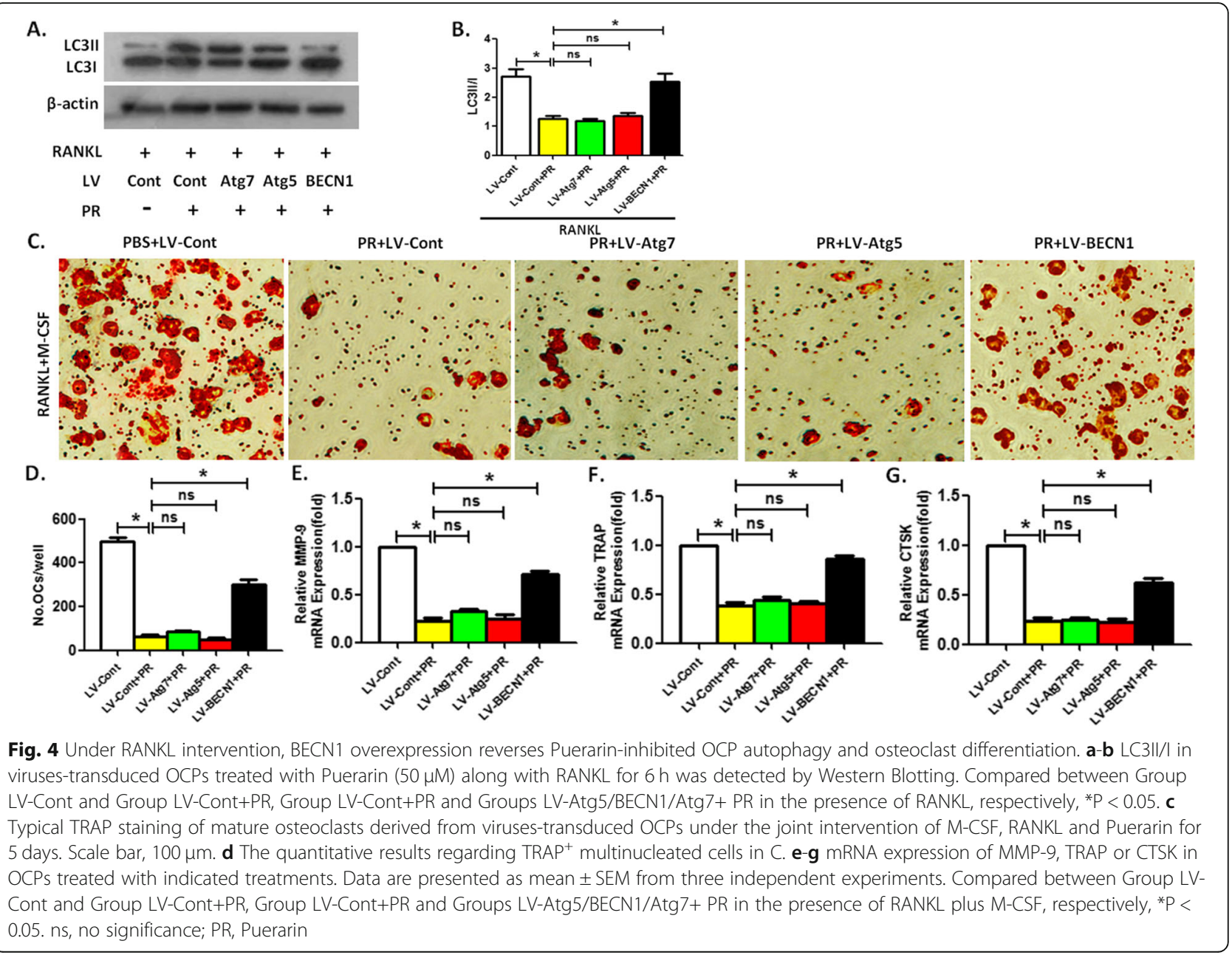




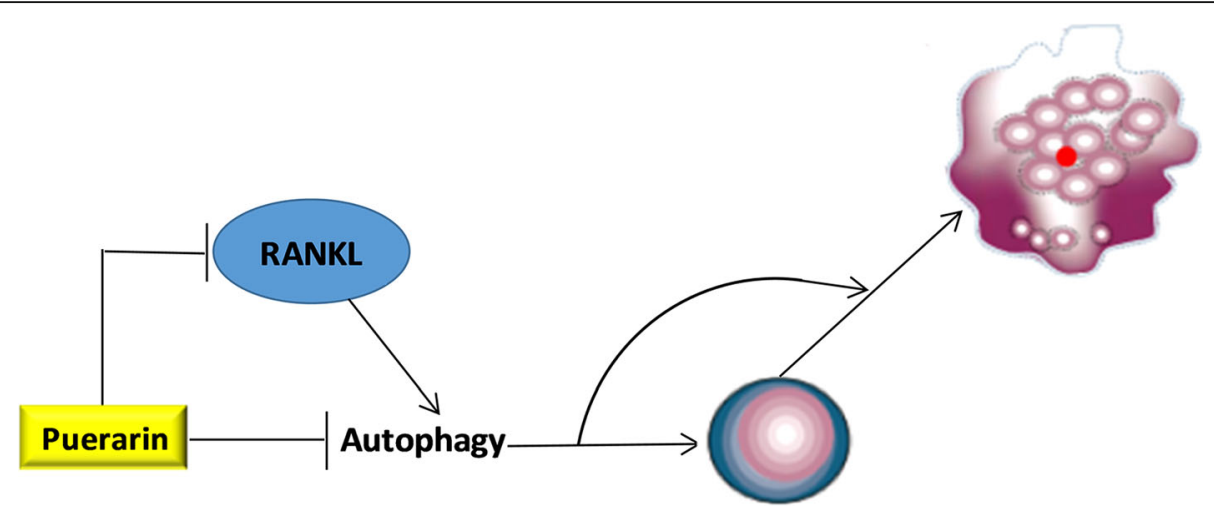

Fig. 5 The current working model regarding Puerarin-regulated autophagy during the osteoclastogenesis

molecular manipulations and classic pharmacological inventions, our findings revealed an important autophagic mechanism underlying Puerarin-treated osteoclastogenesis.

Osteoclast formation is known to consist of the proliferation, fusion and differentiation of OCPs [28]. Firstly, we found that Puerarin directly inhibited the autophagy and proliferation of OCPs in the absence of RANKL, which could be reversed by the overexpression of autophagic genes. This suggested that the direct inhibition of Puerarin on autophagy was responsible for its inhibitory effect on the proliferation of OCPs. It should be noted that overexpression of Atg7 had the strongest effect to reverse Puerarin-inhibited OCP autophagy and proliferation, which was inconsistent with the result that under inducement of RANKL, only BECN1 reversed Puerarin-inhibited osteoclast differentiation. The more research is needed to explore the specific contribution of autophagic genes to OCP proliferation, differentiation and subsequent bone resorption under Puerarin treatment in the future. Then, we observed that Puerarin also inhibited the enhancement of RANKL on OCP autophagy. Moreover, BECN1 overexpression reversed the inhibition of Puerarin on OCP autophagy and osteoclast differentiation. Puerarin not only effectively inhibits the expression of RANKL [29-31], but also blocks RANKLinduced osteoclastogenesis [6], suggesting that Puerarin can inhibit RANKL signaling during the osteoclastogenesis. Therefore, Puerarin prevented RANKL-induced osteoclastogenesis by inhibiting the upregulation of RANKL in OCP autophagy. Moreover, Beclin1 could be regarded as a specific autophagic molecule in Puerarin-treated osteoclastogenesis. Moreover, in the presence or absence of RANKL, the autophagic genes that significantly reversed the inhibitory effect of Puerarin were inconsistent. These results suggested that Puerarin might inhibit OCP autophagy via direct inhibition and inhibiting the signal transduction of RANKL, thereby blocking the formation of osteoclasts. Our current model of Puerarin-regulated autophagy during the osteoclastogenesis is illustrated in Fig. 5.

\section{Conclusion}

Puerarin is well accepted as an autophagy regulator and osteoclastogenesis inhibitor. However, the role of autophagy in Puerarin-regulated osteoclastogenesis is still unclear. The present study clarified the potential mechanism regarding osteoclastogenesis treated by Puerarin from the angle of autophagic response. It proved the effect of Puerarin on inhibiting the autophagic activity of OCPs in the absence or presence of RANKL, which is responsible for Puerarin-inhibited proliferation and differentiation of OCPs respectively. This study not only explored the molecular mechanisms of Puerarin-inhibited osteoclastogenesis, which laid the foundation for further investigation, but also presented novel clues for improving the therapeutic strategies of Puerarin in treating osteoporosis. In addition, the role of autophagy in the resorption activity of the osteoclast treated by Puerarin has not been studied, which suggests research gaps to be bridged in future studies.

\section{Abbreviations}

Atg: Autopahgy related gene; BMMs: Bone marrow-derived macrophages; BSA: Bovine serum albumin; Ligand: LC3Microtubule-associated protein 1 light chain 3; OCPs: Osteoclast precursors; PFA: Paraformaldehyde; RANKL: Receptor activator for nuclear factor-kB; TEM: Transmission electron microscopy; TRAP: Tartrate resistant acid phosphatase

\section{Acknowledgements}

Not applicable.

\section{Authors' contributions}

YZM conceived and designed the experiments; GYZ and YW performed the experiments; GYZ and GKT analyzed the data; YZM and GYZ wrote the manuscript. All authors read and approved the final manuscript.

\section{Funding}

This work was supported by The National Natural Science Foundation of China (81371915), which supported the study design and data collection.

\section{Availability of data and materials}

The datasets used and analyzed in the current study are available from the corresponding author on reasonable request. All data generated or analyzed during this study are included in this published article. 


\section{Ethics approval and consent to participate}

The experimental protocols regarding the extraction of mice BMMs were approved by the Institutional Animal Care and Use Committee of Southern Medical University.

\section{Consent for publication}

Not applicable.

\section{Competing interests}

The authors declare that they have no competing interests.

\section{Author details}

'Department of Orthopedics, Southern Medical University, Guangzhou 510515, Guangdong, China. ${ }^{2}$ Department of Orthopaedics, The 8th medical center of chinese PLA general hospital, No. 17 Heishanhu Road, Haidian District, Beijing 100091, China. ${ }^{3}$ Department of Orthopaedics, Inner Mongolia Tongliao City Hospital, Tongliao 028000, Inner Mongolia, China. ${ }^{4}$ Department of Orthopaedics, Chifeng Hospital, Chifeng 024000, Inner Mongolia, China. ${ }^{5}$ Department of Orthopaedics, The affiliated Zhuzhou hopital XiangYa medical college CSU, Zhuzhou 412000, Hunan, China.

Received: 26 June 2019 Accepted: 20 September 2019

Published online: 15 October 2019

\section{References}

1. Fu R, Zhang Y, Guo Y, Zhang Y, Xu Y, Chen F. Digital gene expression analysis of the pathogenesis and therapeutic mechanisms of ligustrazine and puerarin in rat atherosclerosis. Gene. 2014;552:75-80.

2. Cho HJ, Jun HJ, Lee JH, Jia Y, Hoang MH, Shim JH, Park KH, Lee SJ. Acute effect of high-dose isoflavones from Pueraria lobata (Willd.) Ohwi on lipid and bone metabolism in ovariectomized mice. Phytother Res. 2012;26:1864-71.

3. Guo CJ, Xie JJ, Hong RH, Pan HS, Zhang FG, Liang YM. Puerarin alleviates streptozotocin (STZ)-induced osteoporosis in rats through suppressing inflammation and apoptosis via HDAC1/HDAC3 signaling. Biomed Pharmacother. 2019;115:108570.

4. Urasopon N, Hamada Y, Cherdshewasart W, Malaivijitnond S. Preventive effects of Pueraria mirifica on bone loss in ovariectomized rats. Maturitas. 2008:59:137-48.

5. Park KH, Gu DR, Jin SH, Yoon CS, Ko W, Kim YC, Lee SH. Pueraria lobate inhibits RANKL-mediated Osteoclastogenesis via Downregulation of CREB/ PGC1ß/c-Fos/NFATc1 signaling. Am J Chin Med. 2017;45:1725-44.

6. Yuan SY, Sheng T, Liu LQ, Zhang YL, Liu XM, Ma T, Zheng H, Yan Y, Ishimi Y, Wang XX. Puerarin prevents bone loss in ovariectomized mice and inhibits osteoclast formation in vitro. Chin J Nat Med. 2016;14:265-9.

7. Zhang Y, Yan M, Yu QF, Yang PF, Zhang HD, Sun YH, Zhang ZF, Gao YF. Puerarin prevents LPS-induced osteoclast formation and bone loss via inhibition of Akt activation. Biol Pharm Bull. 2016;39:2028-35.

8. Lin NY, Beyer C, Giessl A, Kireva T, Scholtysek C, Uderhardt S, Munoz LE, Dees C, Distler A, Wirtz S, Krönke G, Spencer B, Distler O, Schett G, Distler $\mathrm{JH}$. Autophagy regulates TNFa-mediated joint destruction in experimental arthritis. Ann Rheum Dis. 2013;72:761-8.

9. Lin NY, Chen CW, Kagwiria R, et al. Inactivation of autophagy ameliorates glucocorticoid-induced and ovariectomy-induced bone loss. Ann Rheum Dis. 2016;75:1203-10

10. DeSelm CJ, Miller BC, Zou W, Beatty WL, van Meel E, Takahata Y, Klumperman J, Tooze SA, Teitelbaum SL, Virgin HW. Autophagy proteins regulate the secretory component of osteoclastic bone resorption. Dev Cell. 2011;21:966-74

11. Zhou XL, Wan XM, Fu XX, Xie CG. Puerarin prevents cadmium-induced hepatic cell damage by suppressing apoptosis and restoring autophagic flux. Biomed Pharmacother. 2019;115:108929.

12. Liu B, Wu Z, Li Y, Ou C, Huang Z, Zhang J, Liu P, Luo C, Chen M. Puerarin prevents cardiac hypertrophy induced by pressure overload through activation of autophagy. Biochem Biophys Res Commun. 2015;464:908-15.

13. Wang LY, Fan RF, Yang DB, Zhang D, Wang L. Puerarin reverses cadmiuminduced lysosomal dysfunction in primary rat proximal tubular cells via inhibiting Nrf2 pathway. Biochem Pharmacol. 2019;162:132-41.

14. Hongyun H, Tao G, Pengyue Z, Liqiang Y, Yihao D. Puerarin provides a neuroprotection against transient cerebral ischemia by attenuating autophagy at the ischemic penumbra in neurons but not in astrocytes. Neurosci Lett. 2017;643:45-51.
15. Wang JF, Mei ZG, Fu Y, Yang SB, Zhang SZ, Huang WF, Xiong L, Zhou HJ, Tao W, Feng ZT. Puerarin protects rat brain against ischemia/reperfusion injury by suppressing autophagy via the AMPK-mTOR-ULK1 signaling pathway. Neural Regen Res. 2018;13:989-98.

16. Tang $H$, Song $X$, Ling $Y$, Wang $X$, Yang $P$, Luo $T$, Chen A. Puerarin attenuates myocardial hypoxia/ reoxygenation injury by inhibiting autophagy via the Akt signaling pathway. Mol Med Rep. 2017;15:3747-54.

17. Ji L, Gao J, Kong R, Gao Y, Ji X, Zhao D. Autophagy exerts pivotal roles in regulatory effects of 1a,25-(OH)2D3 on the osteoclastogenesis. Biochem Biophys Res Commun. 2019;511:869-74.

18. Klionsky DJ, Abdelmohsen K, Abe A, Abedin MJ, Abeliovich H, Acevedo Arozena A, Adachi H, et al. Guidelines for the use and interpretation of assays for monitoring autophagy (3rd edition). Autophagy. 2016;12:1-222.

19. Ke D, Ji L, Wang Y, Fu X, Chen J, Wang F, Zhao D, Xue Y, Lan X, Hou J. JNK1 regulates RANKL-induced osteoclastogenesis via activation of a novel $\mathrm{BCl}-2$ Beclin1-autophagy pathway. FASEB J. 2019;33:11082-11095.

20. Sun KT, Chen MY, Tu MG, Wang IK, Chang SS, Li CY. MicroRNA-20a regulates autophagy related protein-ATG16L1 in hypoxia-induced osteoclast differentiation. Bone. 2015;73:145-53.

21. Lu X, Su N, Yang J, Huang W, Li C, Zhao L, He Q, Du X, Shen Y, Chen B, Chen $\mathrm{L}$. Fibroblast growth factor receptor 1 regulates the differentiation and activation of osteoclasts through Erk1/2 pathway. Biochem Biophys Res Commun. 2009;390:494-9.

22. Ha J, Choi HS, Lee Y, Kwon HJ, Song YW, Kim HH. CXC chemokine ligand 2 induced by receptor activator of NF-kappa B ligand enhances osteoclastogenesis. J Immunol. 2010;184:4717-24.

23. Karakashev S, Zhu H, Wu S, Yokoyama Y, Bitler BG, Park PH, Lee JH, Kossenkov AV, Gaonkar KS, Yan H, Drapkin R, Conejo-Garcia JR, Speicher DW, Ordog T, Zhang R. CARM1-expressing ovarian cancer depends on the histone methyltransferase EZH2 activity. Nat Commun. 2018;9:631.

24. Saiki S, Sasazawa Y, Imamichi Y, Kawajiri S, Fujimaki T, Tanida I, Kobayashi H, Sato F, Sato S, Ishikawa K, Imoto M, Hattori N. Caffeine induces apoptosis by enhancement of autophagy via PI3K/Akt/mTOR/p70S6K inhibition. Autophagy. 2011;7:176-87.

25. Wang X, Qi H, Wang Q, Zhu Y, Wang X, Jin M, Tan Q, Huang Q, Xu W, Li X, Kuang L, Tang Y, Du X, Chen D, Chen L. FGFR3/fibroblast growth factor receptor 3 inhibits autophagy through decreasing the ATG12-ATG5 conjugate, leading to the delay of cartilage development in achondroplasia. Autophagy. 2015;11:1998-2013

26. Xiu Y, Xu H, Zhao C, Li J, Morita Y, Yao Z, Xing L, Boyce BF. Chloroquine reduces osteoclastogenesis in murine osteoporosis by preventing TRAF3 degradation. J Clin Invest. 2014;124:297-310

27. Chung $Y$ H, Jang $Y$, Choi B, Song DH, Lee EJ, Kim SM, Song Y, Kang SW, Yoon SY, Chang EJ. Beclin-1 is required for RANKL-induced osteoclast differentiation. J Cell Physiol. 2014;229:1963-71.

28. Boyle WJ, Simonet WS, Lacey DL. Osteoclast differentiation and activation. Nature. 2003;423:337-42

29. Wang Y, Yang C, Xie WL, Zhao YW, Li ZM, Sun WJ, Li LZ. Puerarin concurrently stimulates osteoprotegerin and inhibits receptor activator of NF-KB ligand (RANKL) and interleukin-6 production in human osteoblastic MG-63 cells. Phytomedicine. 2014;21:1032-6.

30. Yang X, Zhang H, Wang J, Zhang Z, Li C. Puerarin decreases bone loss and collagen destruction in rats with ligature-induced periodontitis. J Periodontal Res. 2015;50:748-57.

31. Yan B, Zhou H, Chu J, Cao X. Suppression of puerarin on polymethyImethacrylate-induced lesion of peri-implant by inhibiting NF-KB activation in vitro and in vivo. Pathol Res Pract. 2019:1:152372.

\section{Publisher's Note}

Springer Nature remains neutral with regard to jurisdictional claims in published maps and institutional affiliations. 